\title{
Special Section Guest Editorial: Quality Control by Artificial Vision VI
}

\author{
Christophe Cudel, ${ }^{a}$ Oliver Aubreton, ${ }^{b}$ Kazunori Umeda, ${ }^{c}$ and \\ Kunihito Kato ${ }^{\text {d }}$ \\ aUniversité de Haute Alsace, IRIMAS Research Institute, Mulhouse, France \\ ${ }^{\text {b} U n i v e r s i t e ́ ~ d e ~ B o u r g o g n e, ~ I m V i A ~ R e s e a r c h ~ G r o u p, ~ L e ~ C r e u s o t, ~ F r a n c e ~}$ \\ ${ }^{\mathrm{c}}$ Chuo University, Department of Precision Mechanics, Tokyo, Japan \\ ${ }^{\mathrm{d}}$ Gifu University, Faculty of Engineering, Gifu, Japan
}

The Special Section on Quality Control by Artificial Vision VI focuses on the latest research works in the field of control and inspection by vision. The special section was suggested by participants of the 2019 International Conference on Quality Control by Artificial Vision (www.qcav2019.uha.fr). The 13 accepted papers cover all the main topics of quality control, such as nonconventional acquisition, 3-D vision, and deep learning applied to the inspection tasks for industry, but also some in the medical imaging field.

On the medical side, the papers of this special section concern skin inspection. Abubakar et al. propose a machine learning for human skin burns classification, and El Kabir et al. developed an interesting approach to graduate the degree of psoriasis.

In nonconventional acquisition techniques, Miazaki et al. estimate the shape of concave specular objects by using the properties of the polarization of surface through some different points of view. In the field of polarization, Gimenez et al. present a complete survey of calibration algorithms for polarization filter array, and Nagorny et al. propose an original approach to exploit the polarimetric imaging for controlling the surfaces of pieces produced by plastic injection. Concerning the works of Castro et al., they describe the interaction between surfaces and light and propose a method to improve the estimation of the real spatial distribution of the lighting directions for acquisition according to the reflectance transformation imaging (RTI) technique.

In the field of 3-D point clouds, Ben Abdallah et al. propose an interesting work for checking the wiring interconnection on an aircraft, based on a dataset of 3-D points obtained with 3-D scanners. They can reconstruct a 3-D model of the wiring harnesses and the results are very promising for any industrial inspection. Mikhailov et al. present the classification of 3-D point clouds for the automatic inspection of complex aeronautical mechanical assemblies.

Belhadj et al. describe the problem of the detection of transparent and foreign objects in semiopaque bottles. This work uses the kurtosis and local information that emphasizes connected components.

Two contributions present the problem of the detection of defects on some reflective surfaces, such as metallic objects. Both used the deflectometry and convolutional neural network approaches. Fantinel et al. develop the residual of linear evolution of light (RLEL) combined with a hybrid learning technique for detecting faulty surfaces. In their paper, Maestro-Watson et al. explore the use of a fully convolutional neural network to perform semantic segmentation of deflectometry recordings. The results are highly correlated with the real impact observed on each defect.

On the reflective surface, deep learning shows also its interest in performing efficient inspections in the industrial environment. Goto et al. used an adversarial autoencoder for detecting anomalies on printed circuit boards. Miranda et al. propose a full scan of a fuselage aircraft thanks to a camera embedded on aerial unnamed vehicles. They have developed an original approach, based on a hybridization of deep and prototypical neural networks for inspecting the content of the corresponding images.

All these papers show that the quality control by vision is a rich research area, combining the best of the state of the art not only in acquisition techniques but also in machine and deep learning.

(ㄱ) 2020 SPIE and IS\&T 
Christophe Cudel received his $\mathrm{PhD}$ degree in 1995. He is a full professor at the Université de Haute Alsace. His current research is on light field cameras for quality control and visual navigation. He is involved in several projects with industrial companies and academic partners. $\mathrm{He}$ was the main organizer of the internal conference QCAV in 2019.

Olivier Aubreton ( $\mathrm{PhD}$ '04) is currently an assistant professor in machine vision and computer vision. He is currently working on the development of solutions based on IR and polarimetric imaging for 3-D reconstruction of specular/transparent surfaces, nondestructive testing (NDT), and quality control by vision.

Kazunori Umeda received $\mathrm{BS}$, MS, and $\mathrm{PhD}$ degrees in precision mechanics from the University of Tokyo, Japan, in 1989, 1991, and 1994, respectively. He became a lecturer in the Department of Precision Mechanics at Chuo University, Japan, in 1994, and is currently a professor since 2006. He was a visiting worker at National Research Council of Canada from 2003 to 2004. His research interests include robot vision, 3D vision, and human interface using vision.

Kunihito Kato is an associate professor at Gifu University of Faculty of Engineering. He received his BS and MS degrees and his $\mathrm{PhD}$ from Chukyo University of Faculty of Information Science in 1993, 1995, and 1996, respectively. He has been a faculty member at University of Maryland Institute for Advanced Computer Studies. His research interests include image processing, pattern analysis, and computer vision. 